\title{
Development of autonomous bio-production vehicle for agriculture
}

\author{
Wan Ishak Wan Ismail ${ }^{1,2}$, Yong Win Loon ${ }^{2}$ and Mohd. Hudzari Razali ${ }^{1}$ \\ ${ }^{1}$ Institute of Advance Technology and ${ }^{2}$ Faculty of Engineering, 43400, Universiti Putra Malaysia, \\ Serdang, Selangor, Malaysia, wiwi@eng.upm.edu.my, hud47@hotmail.com
}

Abstract- The study was carried out to automate the operation of the 'Bobcat' tractor for the farming purpose. Bobcat tractor is a hydrostatic tractor that uses levers for steering, breaking and the forward and backward movements. Two set of screw shaft driven by car power window motor were used to move the two levers. The computer program was written to automate the movement of each lever. Bobcat tractor move straight forward when both levers move forward and vice versa. Bobcat tractor will turn left when only the left lever is moved forward and vice versa. The friction between the levers and the attachment was a major concern in this project. The bearing was introduced and the existence space between the levers and the attachment can solve the friction problem. Autonomous tractor increases the effectiveness of the field with little or no help of supervision.

Keywords; Bobcat Tractor, Autonomous Tractor, Bio-Production Vehicle, Hydrostatic drive, Lever steering, Master-Slave Tractor

\section{Introduction}

Traditional agriculture consumes lots of labor force and induces high cost. Furthermore, manual operation is not effective with the current available technology nowadays. These are the factors that affect the development of agriculture in our country. A new technology is needed to decrease the labor force and increase the efficiency during production (Wan Ishak, 2007). In recent years, a common way to reduce labor force is by replacing the labor force with new and advanced technology. Automation in agriculture processes is more economical, efficient and reliable than human labor. However, automation in agriculture is still in an early stage of development because of the complex challenges (Wan Ishak and Hudzari, 2009). The most common problem faced is the safety issues that may affect the immediate surroundings. Nevertheless, agricultural automation bears great promise for the future both economically and environmentally. (Reske-Nielsen et la., 2006). In western country, huge tractor is used to increase the efficiency of the work in the big scale of land. However agriculture lands in Malaysia are in smaller scale, and the soil structure is softer. Thus, huge tractor cannot be employed in our land due to the soft soil which could lead to tractor bogging down and soil damage. The compact soil may decrease the productivity of the crops (Mejnertsen, A. and Reske-Nielsen, 2006). A small tractor, which is the lighter equipment that prevents soil damage, is more suitable in our country. However, a small tractor alone cannot do the job efficiently therefore a numbers of small tractors are required to increase the efficiency. A new technology, which is a master-slave system comprised of several agriculture tractors, is introduced to replace the huge tractor (O'Connor at el., 1996). A numbers of automated slave tractors are following the command from the master tractor while the master tractor receives command from the operator. The slave tractors will copy the movement of the master tractor and move as the master tractor move. A system for autonomous tractor will be developed first before setting up the communication system between master and slave tractor. The autonomous tractor gives the advantage that the driver can control the tractor from indoor. Hence, the bad weather condition will no longer become a barrier to operate the tractor. To fully automate tractor is a great challenge to the engineer. The autonomous tractor can be further developed to the master-slave system (Wan Ishak, 2009). Usually, an autonomous tractor is able to operate without a driver. However, the difficulty to develop automated tractor increases when the tractor is operated in the changing environment (Brown et al., 1990). The first step to automate the tractor is to automate the steering of the tractor. Therefore, this study is mainly focused on the automation of the tractor's steering.

The primary objective of the study is to modify the Bobcat tractor into an autonomous tractor for farming operation. The Bobcat tractor is a skid steering vehicle with the hydrostatic drive. "The skid steering vehicle is turned by generating differential velocity at the opposite sides of the vehicle, as the wheels or tracks are on the fixed position." The steering system of the Bobcat tractor is controlled by two levers, unlike normal vehicle is controlled by a steering wheel. A module was developed to work as the brain of the tractor to receive data and give command to the tractor. A user-friendly GUI was developed to enable the ease of control the tractor by users. Another program was developed to link up the GUI and the modules. The command given to the tractor can be done by using computer. The movement of the tractor can be controlled by using the computer.

\section{LITERATURE REVIEW}

Several studies on the autonomous tractor were carried out (Zhang et al., 1999; Saufi et al., 2007; Wilson 2009) Automatic guidance system is considered as one of the most appealing autonomous tractor. With the help of the operator, the tractor is given a desired position by using computer. This desired 
position is compared with the measured position by the use of the position sensor. With the difference between the desired and measured position, the steering controller calculate steering control signal and send it to drive the steering actuator. The steering actuator continues to rotate until the tractor reaches the desired position. By having the feedback element, the autonomous tractor is possible. (Wilson, 2000)

There are a number of researches conducted on the topic of autonomous tractor. An automatic tractor with the use of global position system (GPS) to control the movement was developed in a research project at Stanford University. This research shows that a tractor in a field can be controlled by using only GPS. The movement position has a very high degree of accuracy. A manually driving path was initially carried and it works as a reference path for the tractor. The tractor moved base on that reference path. Thus, this encounter problem when there is a sudden change in environment. The tractor will hit obstacle when there is moving object appeared in environment. (O'Connor et al., 1996)

A combine harvester with the use of local information for navigation was developed at University of Delaware. The movement of the combine harvester was controlled by machine vision. The accuracy of the system is as high as the best GPS system. The combine harvester can harvest the corns in a more precise way by the aid of camera. The disadvantage of this system is the vision algorithm that encounters problems when the combine harvesters shadow blocks the camera. The camera also only works in near optimal lighting conditions and the result might be different if the environment is not in optimum condition. (Benson et al., 2001).

A robotic harvester, called Dememter, has been developed for automated harvesting of windrowed crops. Sensor fusion is introduced to automate this robotic harvester. Sensor fusion is the process of combining data from multiple sensors. The robot platform was a New Holland 2550 self-propelled windrower equipped with DGPS, inertial navigation system (INS), and two cameras. (Pilarski et al., 1999)

A research project of unmanned tractor had been carried out at University Putra Malaysia. The paper shows the basic concepts of developing an autonomous tractor. The modification of electromechanical was done on the auto ignition system, acceleration system, gear system, breaks system, and steering system. The controller that was used on this tractor is ICPCON-7000. The program and GUI were designed for the controller of the tractor. (Saufi, 2002).

This project will focus on the autonomous skidsteering tractor for the master-slave system. The research work will be a part of a research project within the field of master-slave system. The hardware was compatible with the software to convert the electrical signal to the mechanical motion.

\section{METHODOLOGY \\ General Overview}

Figure 1 shows the bobcat tractor before the modification. The forward and backward movements of the tractor were controlled by using two levers. These two levers are not only used for steering purpose, but also being used in brake system and acceleration system. When two levers are pushed forward, the tractor moves forward and vice versa. The speed of the tractor is determined by the forward position of the levers. The bigger angle of the lever pushed forward, the faster the tractor travel. When both levers are in the middle position, the tractor is stopped. When one lever is pushed forward and one lever is pushed backward, the tractor will turned. When the left hand side lever is pushed forward and the right hand side is pushed backward, the tractor turns left. When the right hand side lever is pushed forward and the left hand side is pushed backward, the tractor turns right. Thus, this study will focus on how to automate these two levers for the purpose of autonomous tractor.

The primary objective was to develop hardware to steer the tractor either to move forward, backward, turn left or turn right. The two levers need to be pushed in opposite direction in order to turn the tractor. A motor only can move both levers in the same direction, which means both levers either move forward or backward. In other word, one motor only drive the tractor move forward or backward but it can't turn the tractor left or right. Thus two motors were used, one motor for each lever. The challenge of this project was to make sure that both levers can move smoothly and easily while the tractor moves. The design consideration took into account of how to synchronize both the motors to control the levers in such a way that it can move forward, move backward, accelerate the movement of tractor, turn left, turn right and to stop the tractor. The other challenge was to write the program to synchronize the movement of the two levers for accurate angle of turn during cornering.

\section{Steering System}

Two set of steering systems were designed to operate these two levers. One set of automated system consists of one motor, and one attachment. The motors that were used to convert electrical energy into mechanical energy are power window motors. Figure 2 shows the motor that was used in this system. The power window motor can use 12 volts battery from the tractor Two motor are used to drive the two levers.

Figure 3 shows an attachment consisted of one power screw and sets of bearing. Power screws are designed to convert rotational movement of the motor into the linear movement. The power screws also exert the 
necessary force to move the levers along a desired path. The advantage of using power screw is to obtain precise axial movements. Precise linear movement is important in this system because the position of the lever will determine the speed of the tractor. The further the lever is pushed forward, the faster the tractor can move. The hydraulic and pneumatic system does not work in this steering system as both systems move the lever from one point to another point and cannot stop the lever in the middle of the distance between two points. The distance adjustment is available by using the screw with function that is able to adjust the acceleration of the tractor.

Two frames were fabricated to level up the screws and made them compatible with the height of the motor. The screws were fastened on the frames by the bolts and nuts. The materials of all the parts are made of mild steel. Bearing is used to reduce resistance of rotation motion between the screw and the parts on the tractor. Two bearings are installed on the both ends of the screw. The metal balls rotate against smooth surface inside a bearing which reduces friction. These balls bear the load and let the rotating screw to move smoothly. Since the ball is in spherical shape, the ball only contacts the surface on a very small area and this smooth the rotational movement. Besides that bearing is also used on the moving part attached to the lever. This also shares the same purpose to reduce the friction so that the lever moves smoothly. Bearings may need adjustment to minimize the effects of wear and tear if the rotational movement is not smooth. An oil lubrication plug is added on the bearing for the purpose of insertion lubricate. Bearing life can last longer when the bearing is kept clean and well-lubricated.

\section{Specification}

Power screws are classified by the geometry of their thread. The thread of power screw that was used in this system is square thread form. The thread is in square cross section area which is shown in the Figure 4 . The benefit of using square thread is that it has a much higher intrinsic efficiency than trapezoidal or acme threads. The nut does not undergo radial pressure or bursting pressure because it lacks thread angle. This also increases the moving part's life. The disadvantage of this thread is the difficulty in machining such a thread. The thread is usually cut with a single-point cutting tool. This technique is much slower and more expensive than using a multi-point cutting tool. This thread is more difficult to cut because of its perpendicular face. The thread cannot carry as much load as a trapezoidal thread because the root of the thread is larger.

With the reference of Figure 4, pitch is the axial distance measured from a point in one thread to the corresponding point on the adjacent thread. Pitch of the thread used is $6 \mathrm{~mm}$. A $6 \mathrm{~mm}$ represents the reciprocal of the number of thread per inch. One complete revolution of the screw will move an object to a distance equal to the pitch of the screw. Therefore, one complete revolution will move a screw with $6 \mathrm{~mm}$ pitch a distance of $6 \mathrm{~mm}$ of an inch into an object. The mechanical advantage of a screw is equal to the circumference of the screw divided by the pitch of the screw. In other word, the smaller the pitch, the bigger the mechanical advantage can be achieved. Major diameter of the screw is $30 \mathrm{~mm}$ and the root diameter of the screw is $24 \mathrm{~mm}$. The pitch diameter is the theoretical diameter between the major and root diameters. The pitch diameter of the screw is $27 \mathrm{~mm}$. One end of the screw is shrunken into a smaller diameter to let it fit to the motor. The connection between the motor and the screw is shortened as short as possible to increase the efficiency. If the efficiency is too low, the movement of the screw might not be smooth.

Figure 5 is the drawing of the screw by using the Autodesk. The dimension of the screw is also shown. From Figure 5, as can be seen, the hole of the ring that attaches the lever is bigger than the diameter of the lever and it is not tightly fit into the lever. The reason of doing so is to reduce the contact area between the lever and the hole and hence reduces the friction in order to move the lever smoothly. The bearing inside the moving part also enable the hole to adjust itself so that the friction is reduced in another way. The length of the screw should be compatible with the movement of the lever. In other word, the length of the screw should be long enough to push the levers to the maximum level.

\section{Data Acquisition System}

Remote modular data acquisition devices that were used in this system were ICPCON I-7000. ICPCON I-7000 as in Figure 6 is a set of modules whose function is to collect and control data. Only two modules from the series were used. They were I-7042 and 1-7520. I7042 is an isolated digital output module and it has 13 channels. Data exchange between the module and the host is maintained in the ASCII format via a bidirectional communication line of the RS-485 standard. Due to the lack of RS485 on the computer, another module converter is used to convert RS-485 from RS232 on the computer. Figure 7 shows the 4 relays were connected to the motor so only 4 channels from I-7042 were used to control the levers. I-7520 is a high-performance low-cost RS-232 to RS-485 converter packaged. One of the unique features is its self tuning circuitry. This permits the unit to auto-detect baud rate and data formats and tune accordingly. With the connection between the modules and the computer, the command from the computer is able to deliver to the relay.

Algorithm

After all the hardware was installed on the tractor, software was developed so that it can 
be compatible with the hardware. Before writing the program, the relay was tested to run in order to know which relay control is suitable for each motor. The relay number 9 controlled the forward movement of the left lever while the relay 12 controlled the backward movement of the right lever. The relay 10 controlled the backward movement of the left lever and the relay 11 controlled the movement of right lever. Visual basic program was used to automate the levers. 'If' structure was used in the program in order to reverse the movement of the levers and move the levers to the neutral position. The levers in the neutral position make the tractor stop. Timer was inserted into the program to adjust the duration of motors' rotation. The longer the motor rotate the longer distance the lever travel. The larger traveling distances indicates the higher acceleration of the tractor. A simple GUI was developed to let the user control the basic movement of the tractor by simply clicking on the command button inside the GUI. Basically, GUI consists of five commands which are forward, backward, left, right, and stop. The forward command is to move the tractor forward by pushing both levers to move forward. The backward command is to move the tractor backward; the left command is to let the tractor turns left and the right command is to let the tractor turns right. All the commands were labeled so that the user can easily know the function of each command.

\section{RESULT AND DISCUSSION}

Figure 8 shows the modification on tractor to move the lever of the tractor. The modification is the combination of Figure 2 motor and Figure 3 screw. The power screw moves the lever either forward or backward slowly. For the safety concern, the slow movement of the lever can ensure the tractor not to accelerate too fast and easy to stop the tractor. Torque of the motor might create unwanted vibration to the shaft that may shake off the nuts from the bolts. The tightness is ensured by inserting washer which can increase the area of contact between the bolt head or nut and the tractor. After that, the motor was test run by connecting it to a battery. The rotational movement of the motor caused the nut to move forward and also pushed the lever move forward. All the movement is in the smooth motion. Due to the revolution per minute of the both motor is almost the same thus the speed of the moving lever is also almost the same. The different movement speed of the levers can caused the tractor to turn and not move forward.

A program was written to move the lever automatically. A user-friendly GUI was created for the convenience of the operator to control the tractor. The test run for the levers was executed to ensure the program function correctly. All the commands performed were based on their own function; the levers were in correct motion according to the command clicked on the computer. Figure 9 shows the
GUI that was created in this system. The time entered is in millisecond. The user needs to convert the time unit before entering the input. If 1 second is the desired value, the input should be 1000 . The time entered was used to determine the acceleration of the tractor. The longer time inputted, the further distance that the lever traveled, the acceleration will be higher. The emergency command was used to stop the motors but not the tractor. The function is to stop the levers if operator is satisfied with the acceleration of the tractor but the tractor still accelerates forward. The stop command reverses the movement of the levers and returns the levers to the neutral position. When the levers are in the neutral position or middle position, the tractor will stop. The exit command is to clear all the function and exit the GUI. The check box relay lets the user to know which relay is function from time to time. In the view of safety, this system need some time to stop the tractor after the stop command is given. This lag time is referred to the time needed for the levers to travel back its neutral position. The further the levers are pushed, the lag time will be longer. This delay might caused the tractor unable to stop in time during the emergency situation. The further study can be focused on the emergency brake system to solve the aboved problem. The other system such as ignition system and shut down system also are essential elements to be implemented in an real autonomous tractor.

Further development is needed to make this Bobcat tractor into a real autonomous tractor and to make this autonomous system more perfect. Further research can be made is to have a machine vision on the tractor. The effect of intensity changing for environment lighting need consider for working in field application (Razali et al., 2008). The machine vision helps the tractor to identify and avoid obstacle such as people, building, tree and etc. This allows the tractor to complete its task with little or no supervision. The GPS is recommended to be installed on the tractor when the tractor is working in field. GPS has a very high degree of precision. With the guidance of the GPS, the tractor can easily detect the position of the crops. The further study can be focused on the ignition system and other system. If all of the systems recommended are carried out successfully, the autonomous Bobcat tractor can increase the bioproduction in the agricultural sector. A real autonomous tractor does not need any supervision and it can work 24 hours. Hence, the productivity of the crops can be increased in this manner (Wan Ishak, 2009). 


\section{CONCLUSION}

The Bobcat tractor was modified into an autonomous tractor. Based on the engineering knowledge, the design of the power screw was drawn by using Autodesk. Power screw was designed and fabricated to steer the tractor for the purpose of automating the tractor. The thread of the screws was chosen as square because it is the most common thread and has the highest efficiency. The motor rotates the power screw and the rotational movement of the power screw drives the nut to move linearly. Linear movement of the nut pulls the lever to move along the desired path. The two levers are moved smoothly by the rotational of the motor.

The autonomous system was developed to steer the movement of the tractor. A Visual Basic program that is compatible with the GUI was developed in this system. The GUI is simple to let the user to control and steer the tractor easily. The motion of the levers response correctly based on the command entered by the user. The time entered by the user can control the acceleration of the tractor. The important of control application also was mentioned in righteous book, Al. Quran. In surah of Al-Ambia verse 69 stated "We said O fire! be thou cool and (a means of) safety for Ibrahim!" (Ali, 1934). Allah Subhanahuwataala ordered the fire not only to become cool but the condition of cool with control the Ibrahim alaihissalam safely in the fire.

\section{REFERENCES}

[1] Ali A.Y. (1934) Surah Al-Ambia, verse 69, pp. 837

[2] Benson E.R., Reid J.F. and Zhang Q. (2001) Machine vision based steering system for agricultural combines.Paper number 011159, 2001 ASAE Annual Meeting.

[3] Brown N. H., Wood H. C. and Wilson J. N. (1990) Optics in Agriculture, Vol. 1379 , pp. $54-68$.

[4] Guo L., Zhang Q., Han S. (2002) Journal of Agricultural Safety and Health. 8(4): 385-396.

[5] Kunt J. and Reintam E. (2004) Soil compaction effect on soil physical properties and the content of nutrients in spring barley (hordeum vulgare I.) and spring wheat (triticum aestivum I.)

[6] Mejnertsen A. and Reske-Nielsen (2006) Control of Autonomous Tractor, Master's Thesis, Technical University of Denmark.

[7] Muhammad Saufi ,Wan Ishak WI, Mohd. Hudzari (2007) International Conference on Control, Instrumentation and Mechatronics Engineering CIM 07. 28-29 May 2007. Johor Bharu, Johor.

[8] Muhammad Saufi Mohd Kassim (2003) Design and development of unmanned tractor for agricultural

Copyright (C) 2010, Bioinfo Publications operation. Master Theisis, Universiti Putra Malysia.

[9] O'Connor, Michael, Thomas Bell, Gabe Elkaim, and Bradford Parkinson. (1996) 3rd International Conference on Precision Agriculture, Minneapolis, MN, June 23-26.

[10] Pilarski T., Happold M., Pangels H., Ollis M., Fitzpatrick K., Stentz A. (1999) Proc. of the 8th International Topical Meeting on Robotics and Remote Systems, April 1999.

[11] Razali M.H., Wan Ismail W.I., Ramli A.R. and Sulaiman M.N. (2008), International Journal of Food Engineering, Volume 4, No. 3, Article 5.

[12] Reske-Nielsen A., Mejnertsen A., Andersen N. A., Ravn O., Nørremark M., Griepentrog H. W. (2006) Proceedings Automation Technology for Off-Road Equipment (ATOE) Bonn, Germany

[13] Wan Ishak Wan Ismail and Mohd Hudzari Razali (2009) Journal Engineering e-Transaction, 4(1), 1420.

[14] Wan Ishak Wan Ismail (2009) Design and Development of Master-Slave Communication System for Farm Tractor, End of Project Report (RUGs, no. 5523442), Univesiti Putra Malaysia.

[15] Wan Ishak Wan Ismail (2007) Journal of The Ingenieur. Board of Engineer Malaysia. 13, 46-54.

[16] Wei J., Rovira-Mas F., Reid J.F., Han S. (2005) Transactions of the ASABE. 48(6): 2389-2397.

[17] Wilson J. N. (2000) Computer and Electronic in Agriculture, 25(1), 3-9

[18] Zhang Q., John F. Reid and Noboru Noguchi (1999) Proceedings of the International Conference on Field and Service Robotics. 13-15 July, UILU. 


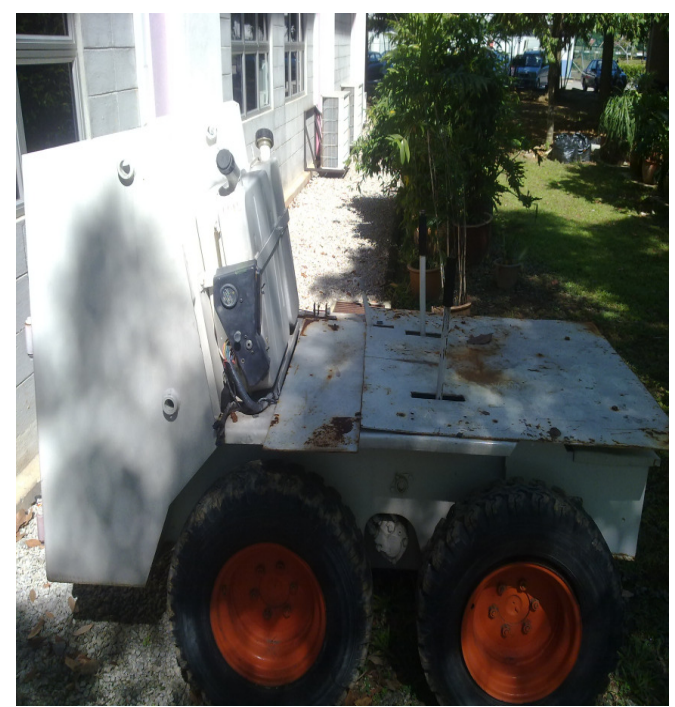

Fig. 1-Bobcat Tractor

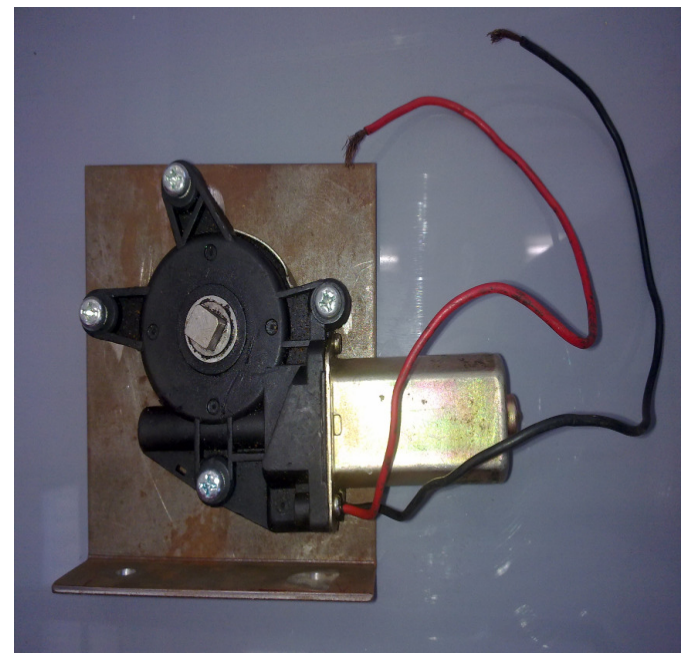

Fig. 2-Power Window Motor

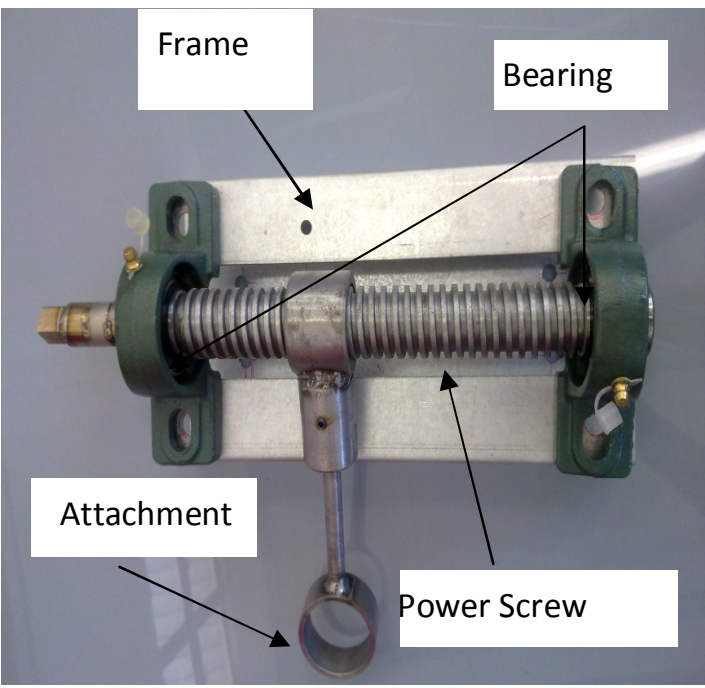

Fig. 3-Power screw with frame

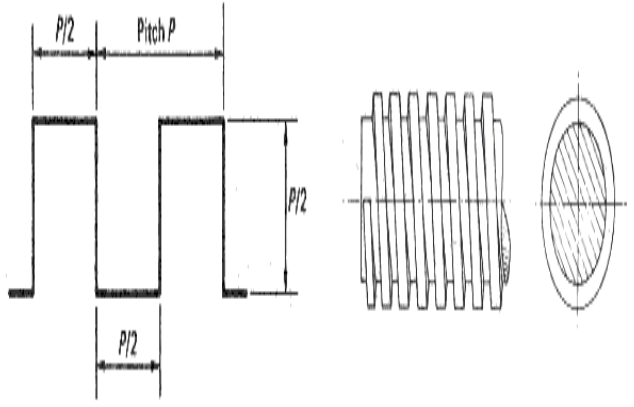

Fig. 4-Square form

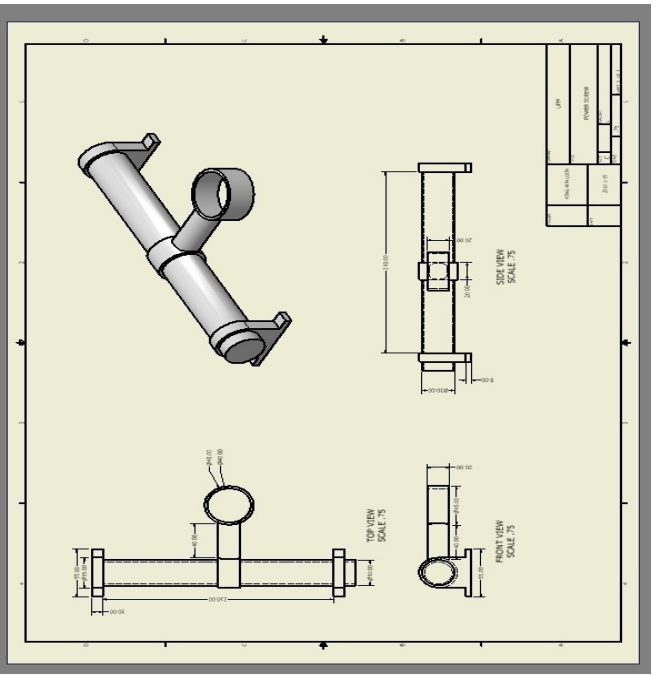

Fig. 5-Drawing of the screw

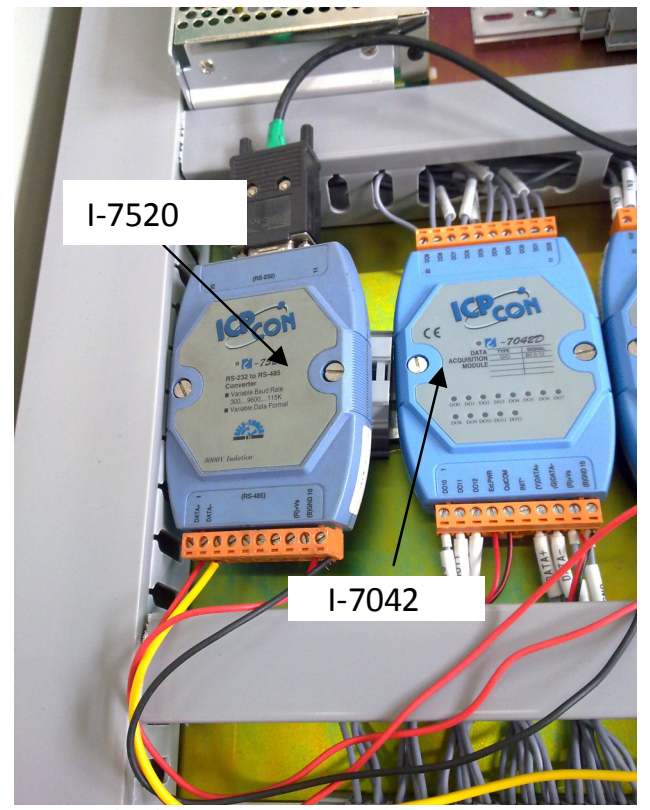

Fig. 6-ICPCON Data Acquisition Hardware 


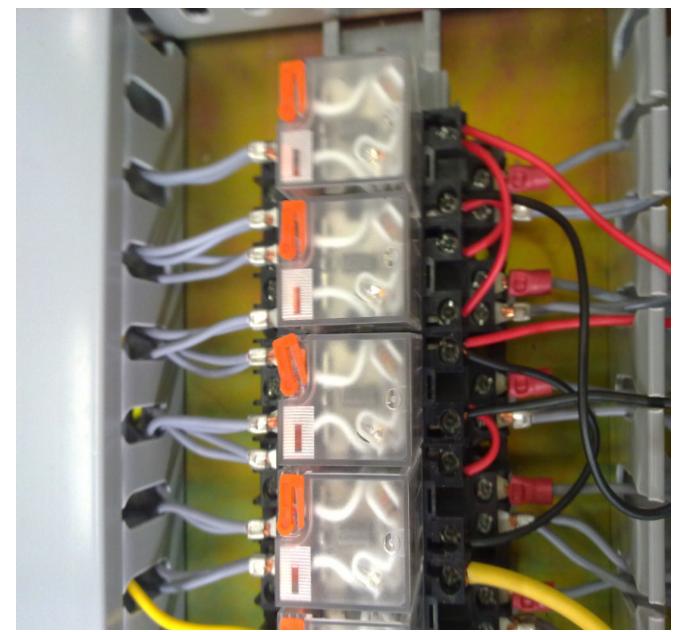

Fig. 7-Relays

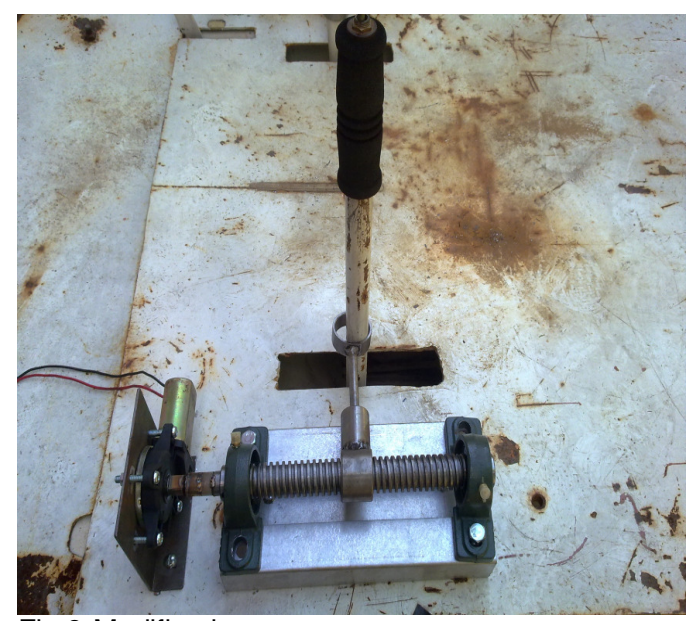

Fig.8-Modification on tractor

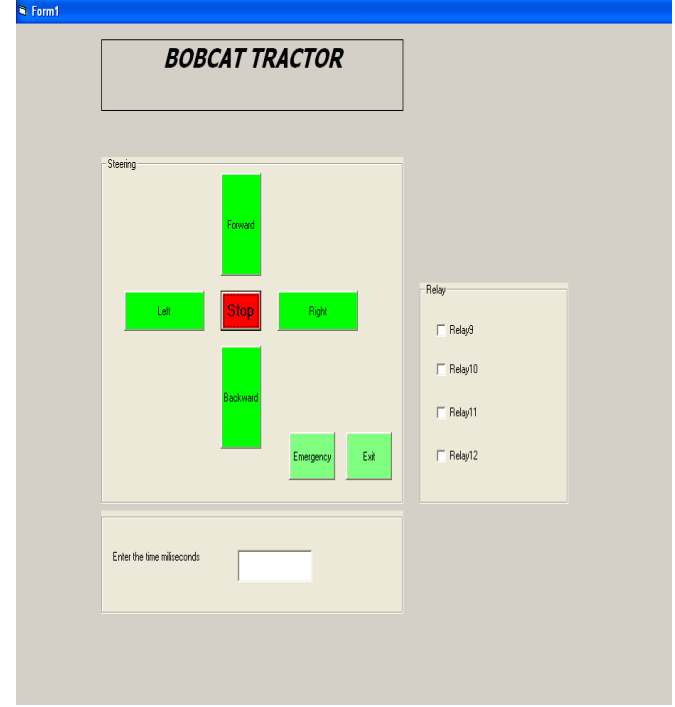

Fig.9-Graphical User Interface 